\title{
Induction with uncertain categories: When do people consider the category alternatives?
}

\author{
Brett K. HaYes and Ben R. Newell \\ University of New South Wales, Sydney, New South Wales, Australia
}

\begin{abstract}
These three experiments examined how people make property inferences about exemplars whose category membership is uncertain. Participants were shown two categories and a novel exemplar with a feature that indicated that the exemplar was more likely to belong to one category (target) than to the other (nontarget). Participants then made categorization decisions and property inferences about the novel exemplar. In some conditions, property inferences could be made only by considering both target and nontarget categories. In other conditions, predictions could be based on both categories or on the target category alone. Consistent with previous studies (e.g., Murphy \& Ross, 1994, 2005), we found that many people made predictions based only on consideration of the target category. However, the prevalence of such single-category reasoning was greatly reduced by highlighting the costs of neglecting nontarget alternatives and by asking for inferences before categorization decisions. The results suggest that previous work may have exaggerated the prevalence of single-category reasoning and that people may be more flexible in their use of multiple categories in property inference than has been previously recognized.
\end{abstract}

Inductive inference involves generalizing information from known to novel exemplars. One powerful form of induction makes use of our understanding of categories as a basis for property inference. For example, if told that a lion has some novel property, people are likely to generalize this property to other members of similar categories, such as tigers, but less likely to generalize to dissimilar kinds, such as swans (Osherson, Smith, Wilkie, López, \& Shafir, 1990; Rips, 1975; Sloman, 1993).

Most research on category-based induction has focused on cases where the category membership of the base or premise exemplar is known with certainty. There are many situations, however, where inductive inferences about an object are required before its category membership has been determined. Consider a person hiking through wilderness who hears rustling in the scrub near her feet. Although the hiker knows of many animals (e.g., snakes, small marsupials, birds) that could make the noise, she cannot be certain about the actual source. Nevertheless, she may wish to make an immediate prediction about how likely it is that the animal is dangerous. In a similar vein, after an initial examination a physician may have several possible diagnoses in mind about what is really troubling a patient. Before a final diagnosis is made, however, the physician may have to make some clinical inferences and suggestions to the patient about managing symptoms.

According to Bayesian models of categorization and inference (e.g., Anderson, 1991; Tenenbaum, Kemp, \& Shafto, 2007), people should consider each of the uncertain category alternatives when making feature infer- ences. Anderson's Rational model, for example, proposes that people make property inferences about objects with uncertain category membership by estimating the probabilities of the predicted property for each category alternative, then combining these estimates and weighting each according to the likelihood of the object being in that category. More precisely, according to the Rational model, the prediction that an object with observed features $F$ has an unobserved feature $j$ involves the computation of a weighted sum across each category $k$ :

$$
P(j \mid F)=\sum_{k} P(k \mid F) \times P(j \mid k) .
$$

So in our hiking example, the conditional probabilities of the animal being dangerous would be estimated for each of the candidate categories of snakes, birds, and so on, and these would be weighted according to the likelihood that the animal comes from each category. Note that in Equation 1 , the probability of the predicted feature $j$ is assumed to be conditionally independent of the observed features $F$ in each category. In other words, the Rational model assumes that people assess the frequencies of the given and predicted features independently, ignoring co-occurrence of feature pairs or higher order combinations of features.

However, a substantial body of evidence suggests that people do not usually reason in this way. Typically, experimental studies of property inference with uncertain categories have found that people ignore uncertainty about category membership, basing their predictions solely on the category to which an object is most likely to belong

B. K. Hayes, b.hayes@unsw.edu.au 
(see Murphy \& Ross, 2007, for a review). According to this view, if the hiker believes that the most likely source of the noise is a snake, she will behave as if it were a snake, with appropriate caution.

The first evidence that people tend to focus on single rather than multiple uncertain categories when making property inferences was reported by Murphy and Ross (1994). In several experiments using artificial stimuli (e.g., geometric shapes, schematic faces, line segments), participants were shown four categories of exemplars (e.g., groups of objects varying in shape and color described as being drawn by four different children). Participants were then presented with a novel exemplar with a given feature (e.g., a cross-shaped drawing) and were asked to predict the other feature (color) most likely to be found in this exemplar. Critically, the category membership of the test exemplar was uncertain. Although one child drew more crosses than any other, and so was the most likely or "target" category, other children drew some crosses, and therefore should have been considered in property predictions. Murphy and Ross (1994) found that people acknowledged that the category membership of test exemplars was uncertain but ignored this uncertainty in property predictions; instead, they consistently made predictions based only on the relative frequency of features within the target category.

The evidence for such "single-category" reasoning has proved quite robust, with replications across a range of inference problems involving artificial and natural categories (e.g., Malt, Ross, \& Murphy, 1995; Murphy \& Ross, 2005; Ross \& Murphy, 1996). It is closely paralleled by another consistent finding: When an object belongs to more than one category (e.g., a muffin is both a kind of bread and a kind of breakfast food), most people consider only one of these categories when making property inferences (Ross \& Murphy, 1999).

\section{Mechanisms Underlying Single-Category Reasoning}

One explanation for single-category reasoning is that people do not understand the conditional nature of problems involving uncertainty. They fail to realize that uncertainty at an earlier stage should have an impact on predictions or choices made at later stages of a problem (cf. Tversky \& Kahneman, 1973, 1981). This tendency is most clearly observed in two-stage gamble problems, in which a final prospect is offered only if participants "win through" to the second stage of a gamble. Tversky and Kahneman (1981) have shown that people usually fail to incorporate first-stage probabilities into risk calculations; instead, they reason as if winning at the first stage were a certainty, creating attractive but nonetheless pseudocertain prospects at the second stage. This neglect of alternative outcomes of the different stages of a gamble can be seen as analogous to the failure to encode information from all of the candidate categories in category-based induction. In this respect, single-category reasoning could be thought of as another example of the many well-documented failures of normative reasoning, such as the pseudocertainty effect, as well as other familiar findings, such as base rate neglect in categorization (e.g., Gluck \& Bower, 1988) and probability judgment (Tversky \& Kahneman, 1973).

This "neglect of uncertainty" account, however, is undermined by demonstrations that, under certain circumstances, people can process property information from multiple categories. This happens when a focus on only one category does not lead to a clear prediction (Murphy \& Ross, 1994). Similarly, strongly associating the property being predicted with a nontarget category can cause the nontarget category to be used in feature prediction. For example, Ross and Murphy (1996) asked people to make predictions about an individual from an uncertain occupational category (most likely a realtor, but it could also have been a burglar). People ignored the nontarget burglar category when making predictions about neutral properties ("Will they ring the doorbell?") but considered it when the property was associated with that category ("Will they check whether the windows are locked?").

To explain these findings, Ross and Murphy (1996) suggested a two-stage account of reasoning with uncertain categories. In Stage 1, when an object is presented for categorization, a number of possible categories to which it might belong are considered, and these are explicitly acknowledged in categorization judgments. In Stage 2, however, typically only information about the most likely category is factored into the computations involved in feature prediction. From a normative point of view, the use of all possible categories increases predictive accuracy, although in practice the processing cost of considering these alternatives may be high, relative to gains in accuracy. Single-category reasoning, like many heuristics, may therefore be viewed as an adaptive compromise between predictive accuracy and cognitive effort.

Critically, this processing account allows for the reactivation of other alternatives during the prediction or choice stage, if attention is specifically drawn to them. The main idea is that people are aware that inferences are being made about exemplars whose category membership is uncertain. However, information from less likely category alternatives will affect property predictions only when attention is specifically drawn to these alternatives during the prediction process.

In this article, we propose and test a modified version of the Ross and Murphy (1996) account. We argue that there are additional routes to promoting the use of information about less likely category alternatives in feature inferences. In particular, we propose that the relative salience of the target and nontarget categories at Stage 1 (categorization) is critical to whether they are used during Stage 2 (property prediction). Experiments 1 and 2 test the prediction that the nontarget category being made more salient during Stage 1 (by highlighting the costs of ignoring the category) should increase the use of that category in subsequent predictions, leading to higher levels of multiple-category reasoning. Experiment 3 examines a related issue - namely, that making an explicit categorization decision at Stage 1 can, in itself, draw people's attention toward the target category (and away from the nontarget), with the result that people rely mainly on the target for predictions. The corollary is that if explicit cat- 
egorization is not required before prediction, we should expect higher levels of multiple-category reasoning.

\section{Increasing Category Salience via Response-Cost Instructions}

One way of increasing attention to nontarget categories during the early stages of reasoning with uncertain categories is to emphasize the cost of ignoring those categories. In previous studies, the cost in predictive accuracy of ignoring nontarget categories has often been small. In many of the Murphy and Ross (1994) studies, for example, the extra effort involved in considering category alternatives led to only a small change in the probability of predicting a particular feature (around $10 \%$ to $15 \%$ ).

In Experiments 1 and 2, we drew attention to nontarget categories through "response-cost" instructions that implied that inattention to these categories would have more serious consequences for the decision maker. For example, in a scenario where the candidate categories were presented as diseases, participants were told that the target category was a relatively minor, easily treatable illness, whereas the nontarget category was serious and possibly terminal. Analogous interventions have been shown to be effective in increasing the consideration of alternative hypotheses in decision making (e.g., Koehler, 1994; Lord, Lepper, \& Preston, 1984; McKenzie, 1998; Trope \& Mackie, 1987). Lord et al., for example, found that when people were asked to test a specific hypothesis about social category membership (e.g., whether an unfamiliar person was an extrovert), they selected only questions that would confirm this hypothesis and neglected the alternative social category (introvert). This bias was largely overcome when people were reminded of the alternative category (e.g., by presenting a profile of a typical introvert) before hypothesis testing. Markman, Baldwin, and Maddox (2005) have also shown that categorization strategies can be altered by varying the relative payoffs and costs associated with classifying the members of different categories. On the basis of these findings, we predicted that when people were given "response-cost" instructions while studying uncertain categories, they would be more likely to consider multiple categories when making subsequent property predictions.

\section{EXPERIMENT 1}

The aim of this experiment was to examine the impact on the use of single- and multiple-category reasoning of instructions that highlight the cost of neglecting less likely alternatives. For this purpose, we developed two induction conditions. The divergent prediction condition followed a design similar to that developed by Murphy and Ross (2009), such that a focus on the target category alone led to a different feature prediction than if both categories were considered. This type of item is illustrated in Table 1. If you were told that a novel patient had a "headache," the target category for this patient would be Terragaxis, because this category has more cases with this symptom. If you were then asked to predict what other symptom this patient had, your prediction would depend on whether you looked at both categories (a multiple-category prediction) or just the target (a single-category prediction). Looking at both disease categories, "sore gums" is most likely to be the predicted feature. Applying Equation 1,

$P($ sore gums $\mid$ headache $)=$

$[P($ Terragaxis $\mid$ headache $) \times P($ sore gums $\mid$ Terragaxis $)]$

$+[P($ Buragamo $\mid$ headache $) \times P($ sore gums $\mid$ Buragamo $)]$

$=[(7 / 11) \times(3 / 8)]+[(4 / 11) \times(6 / 8)]=.51$.

By comparison,

$$
\begin{aligned}
P(\text { rash } \mid \text { headache })= & {[(7 / 11) \times(4 / 8)] } \\
& +[(4 / 11) \times(0 / 8)]=.32 .
\end{aligned}
$$

Table 1

\begin{tabular}{|c|c|c|c|c|c|}
\hline \multicolumn{3}{|c|}{ Terragaxis [Target] } & \multicolumn{3}{|c|}{ Buragamo [Nontarget] } \\
\hline Exemplar & Symptom 1 & Symptom 2 & Exemplar & Symptom 1 & Symptom 2 \\
\hline \multicolumn{6}{|c|}{ Divergent Prediction } \\
\hline 1 & Headache & Rash & 1 & Fever & Sore gums \\
\hline 2 & Headache & Sore gums & 2 & Headache & Dizziness \\
\hline 3 & Headache & Rash & 3 & Fever & Sore gums \\
\hline 4 & Headache & Rash & 4 & Headache & Sore gums \\
\hline 5 & Headache & Sore gums & 5 & Fever & Sore gums \\
\hline 6 & Headache & Sore gums & 6 & Headache & Rash \\
\hline 7 & Stomach pains & Rash & 7 & Headache & Dizziness \\
\hline 8 & Headache & Runny nose & 8 & Fever & Sore gums \\
\hline \multicolumn{6}{|c|}{ Nondivergent Prediction } \\
\hline 1 & Headache & Rash & 1 & Headache & Sore gums \\
\hline 2 & Headache & Rash & 2 & Stomach pains & Sore gums \\
\hline 3 & Headache & Rash & 3 & Headache & Sore gums \\
\hline 4 & Headache & Sore gums & 4 & Headache & Runny nose \\
\hline 5 & Fever & Rash & 5 & Fever & Sore gums \\
\hline 6 & Fever & Sore gums & 6 & Headache & Runny nose \\
\hline 7 & Headache & Sore gums & 7 & Stomach pains & Runny nose \\
\hline 8 & Headache & Sore gums & 8 & Stomach pains & Runny nose \\
\hline
\end{tabular}

Examples of the Experimental Categories

Note-The designation of categories as target or nontarget applies when the given feature is "headache." 
If you looked only at exemplars within the target category, however, "rash" would become the most likely feature. According to this single-category approach, the probability of having a rash is .50 , because $4 / 8$ people in the target category (Terragaxis) have a rash, whereas only 3 out of 8 people have sore gums $(P=.375)$.

Throughout our experiments, the proportion of feature choices consistent with the multiple-category approach in the divergent prediction condition was compared with corresponding choices in a nondivergent prediction condition. In the nondivergent condition, the single-category approach did not lead to a clear feature prediction. When faced with the prediction question, people were forced to look outside the target category and make a multiplecategory prediction or guess. This type of item is illustrated in the lower panel of Table 1. Again, the given feature is "headache" and Terragaxis is the target category. Just examining the target category leads to an ambiguous prediction, because two possible symptoms ("rash" and "sore gums") occur with equal frequency. If the less probable or nontarget category were considered, "sore gums" should be predicted. Applying Equation 1,

\section{$P($ sore gums $\mid$ headache $)=$}

$[P($ Terragaxis $\mid$ headache $) \times P($ sore gums $\mid$ Terragaxis $)]$ $+[P($ Buragamo $\mid$ headache $) \times P($ sore gums $\mid$ Buragamo $)]$ $=[(6 / 10) \times(4 / 8)]+[(4 / 10) \times(4 / 8)]=.50$.

This choice probability is greater than any of the conditional probabilities of other symptoms given "headache."

In designing the experimental categories, we tried to eliminate the possibility that people would ignore them and make predictions based on correlations between given and predicted features. Previous work (e.g., Hayes, Ruthven, \& Newell, 2007) suggests that, when given the option to do so, some people follow the nonnormative strategy of ignoring category membership and only examining exemplars that contain a given feature like "headache." They then make a feature prediction based on the relative frequency with which that feature is paired with other features. Although we view this "feature conjunction" strategy as an interesting alternative approach to reasoning under uncertainty, the aim of the present research was to examine the conditions that led to use of single or multiple categories in feature prediction. As a result, all the experimental categories were designed so that their component feature dimensions were statistically independent within and between categories. This meant that examination of feature correlations within or across categories could not produce a clear feature prediction (e.g., in Table 1, divergent condition, the given feature of "headache" was paired equally often with "rash" and "sore gums," both within the target category and across both categories).

Four nondivergent or divergent prediction items were administered to different groups of participants. Two items were always presented with "standard" instructions and two were presented with "response-cost" instructions. Response-cost instructions highlighted the importance of attending to the nontarget category (e.g., for the disease item in Table 1, the nontarget category was described as "serious and potentially fatal"). The order of presentation of the two types of instructions was counterbalanced across participants. Like the authors of many previous studies (e.g., Murphy \& Ross, 1994, 2005), we were interested primarily in how people used category exemplars to make inferences, rather than in how well they learned or remembered the exemplars. Hence, throughout these experiments we used a "decision-only" paradigm, in which all the information required for feature predictions remained visible for the duration of each item.

\section{Method}

Participants. Sixty-four undergraduate psychology students (47 females, 17 males; average age, 19.52 years) participated for course credit.

Materials. The experimental categories used are illustrated in Table 1. All categories contained eight exemplars, with each exemplar composed of values on two feature dimensions. In the nondivergent prediction condition, the stimuli were designed so that clear feature predictions could be made only by considering both the target and nontarget categories. In the divergent prediction condition, consideration of either the target alone or both target and nontarget categories led to different feature predictions. Four items were generated for each prediction condition. Each item had an identical pattern of feature frequencies within and between categories, but the scenario used to explain and label categories and exemplar features was different in each case. The four scenarios covered a range of domains, with categories variously described as different types of diseases, minerals, cars, or visa applicants (see Appendix A for a list of scenario cover stories). ${ }^{1}$

Procedure. Equal numbers of participants were randomly allocated to either the nondivergent or divergent prediction condition. Participants in each condition were presented with four items. For each item, two categories were presented on the top half of a 17-in. computer screen. Exemplars were presented as text statements with a randomly generated two-letter identifier (e.g., "Patient AC"). Exemplars from different categories appeared on opposite sides of the screen under the relevant category label, with left-right location for each category and order of exemplar listing randomized across trials. After $8 \mathrm{sec}$, a novel test exemplar appeared and participants were asked to decide which category this exemplar belonged to (e.g., "Here is a new patient, who we will call Patient X. Patient X has a headache. Given that Patient $X$ has a headache, what kind of disease do you think Patient X has?"). Responses were made by clicking a mouse on one of the category alternatives listed on the screen. Participants also rated their confidence in this judgment by using the mouse to drag a sliding pointer across a 100 -point scale $(0=$ not at all confident; $100=$ very confident). After responses were made, category questions were replaced with prediction questions (e.g., "Given that Patient X has a headache, what OTHER symptom do you think they are most likely to have?") and a slider confidence rating. Predictions were made by clicking the mouse on one of the three (nondivergent prediction) or four (divergent prediction) possible features listed in random order on the bottom of the screen. The reference categories remained on the screen while all judgments were made. The assignment of particular features for the predictions based on multiple or single categories was counterbalanced between participants (e.g., half received a version in which "sore gums" was the predicted feature based on multiplecategory reasoning and "rash" was the predicted feature based on single-category reasoning; the remainder saw a version in which the roles of these features were reversed).

Two items were administered under "standard" instruction conditions and two were administered with "response-cost" instructions that highlighted the importance of attending to the nontarget category. These instructions mentioned the labels of both the target and nontarget categories and provided some additional information about each category. This information always emphasized the relative salience of the nontarget category, either by emphasizing that it was 
associated with more negative outcomes (e.g., a more serious illness) or more positive outcomes (e.g., had higher monetary value) than the target category. For example, in the disease scenario the response-cost instructions read Please Note: The [nontarget disease] is very serious, and possibly terminal. The [target disease] is a relatively minor, easily treatable illness (see Appendix A for standard instructions and Appendix B for response-cost instructions). Half of the participants completed items with response-cost instructions followed by items with standard instructions (response cost first), and the remainder completed the problems in the reverse order (response cost last).

The additional response-cost instructions appeared on the screen $5 \mathrm{sec}$ after the presentation of the categories and remained in view while predictions and categorization judgments were made. The assignment of specific items to the standard and response-cost conditions was counterbalanced across participants, so that each item appeared in each instruction condition on an equal number of occasions. The order of presentation of the two items presented with standard instructions was randomized for each participant, as was the order of the items presented with response-cost instructions. The experiment took around $15 \mathrm{~min}$ to complete.

\section{Results}

Preliminary analyses confirmed that item version (including positive and negative versions of the responsecost items) had no effect on any of the outcome measures. All subsequent analyses were collapsed over this factor.

Target category identification. The target category was generally identified correctly in both the nondivergent prediction and divergent prediction conditions (.97 and .98 , respectively). Overall, confidence in identification of the target category was reasonably high $(M=70.15)$. Confidence ratings were entered into a 2 (prediction condition) $\times 2$ (instructions) $\times 2$ (order of presentation) ANOVA with repeated measures on the second factor. The only effect to reach significance was the main effect of presentation order $\left[F(1,60)=4.09, p=.04, \eta_{\mathrm{p}}^{2}=.07\right]$. People were more confident in identifying the target category for the last two items they encountered $(M=71.07)$ than they were in the first two items $(M=68.28)$, regardless of instruction type.

Feature predictions. Feature prediction analyses were based only on items for which the target category was correctly identified. Feature predictions were coded as consistent with multiple-category reasoning, single-category reasoning, or "other." The proportion of feature predictions based on multiple-category reasoning is given in Figure 1. The proportion of multiple-category predictions in the nondivergent conditions was above a chance value of .33 [standard instructions, $t(29)=6.44, p<.001$; response cost, $t(29)=5.47, p<.001]$. This means that when it was not possible to make an unequivocal prediction based on the target category alone, rather than guess, people used information from the nontarget category. ${ }^{2}$

To compare multiple-category predictions across conditions, these data were entered into a three-way ANOVA (prediction condition $\times$ instructions $\times$ instruction order) with repeated measures on the second factor. Significantly fewer multiple-category predictions were made in the divergent condition than in the nondivergent prediction condition $\left[F(1,60)=25.96, p<.001, \eta_{\mathrm{p}}^{2}=.43\right]$. When those in the divergent group failed to make multiple-category predictions, they almost always made single-category pre-

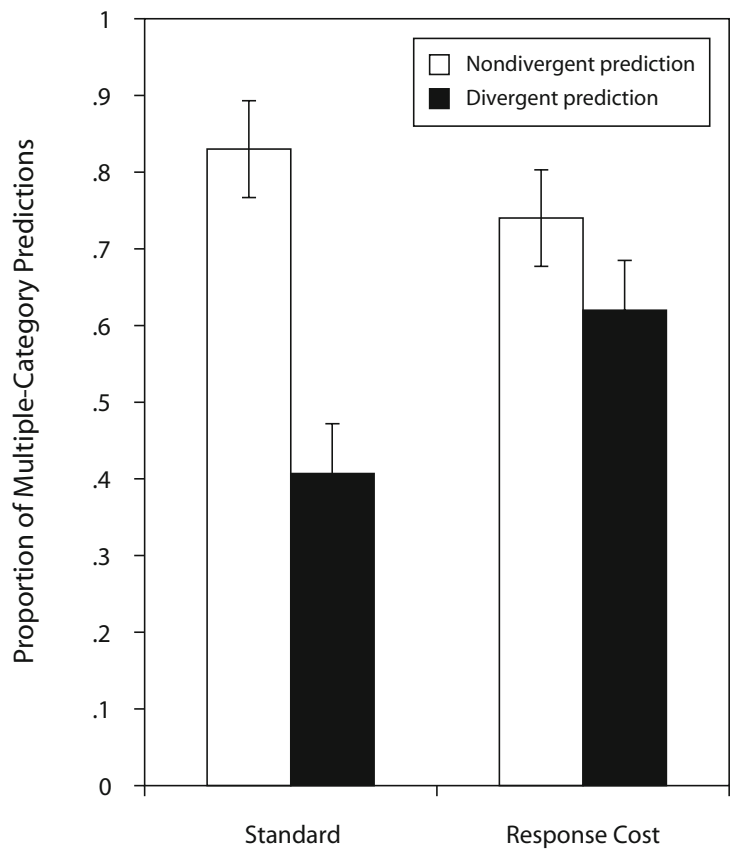

Instructions

Figure 1. Experiment 1: Mean proportion of feature predictions based on multiple categories.

dictions ( $99 \%$ of occasions). In other words, when people had the option of using the target category to make singlecategory predictions, many did so for many items.

This main effect, however, was moderated by an interaction between instructions and prediction condition $\left[F(1,60)=4.02, p=.04, \eta^{2}=.07\right]$. Figure 1 shows that when response-cost instructions were present the proportion of multiple-category predictions in the divergent condition increased relative to the nondivergent condition. Tukey's HSD tests confirmed that people made fewer multiple-category predictions in the divergent condition than in the nondivergent condition when standard instructions were given $(q=4.40, p=.02)$, but that levels of multiple-category prediction did not differ across the prediction conditions when response-cost instructions were given $(q=1.23, p=.2)$.

There were no significant main effects or interactions involving instruction order. In the divergent prediction condition, the impact of response cost on multiple-category responding was unaffected by presentation order $[F(1,60)=$ $0.16]$. Tukey's HSD tests confirmed that response-cost instructions increased the proportion of multiple-category predictions relative to items given standard instructions in both the response-cost-first $(q=3.09, p=.03)$ and response-cost-last $(q=3.34, p<.03)$ conditions. In the divergent condition, there was no increase in multiplecategory predictions for items with standard instructions following completion of items with response-cost instructions $(q=-0.03)$. Hence, there was no indication that the 
effects of response-cost instructions carried over to standard items in the response-cost-first condition.

Confidence in feature predictions was moderate overall $(M=59.63)$. A three-way ANOVA revealed that confidence in predictions in the divergent condition $(M=62.7)$ was higher than in the nondivergent prediction condition $(M=54.11)\left[F(1,60)=4.82, p=.03, \eta_{\mathrm{p}}^{2}=.08\right]$ but was unaffected by instructions or instruction order.

\section{Discussion}

This experiment examined whether people used single or multiple uncertain categories to make property inferences. People readily identified the target category when given a feature of a novel exemplar but acknowledged that category membership of this exemplar was uncertain. When people could only make a feature inference by looking at more than one category (in the nondivergent prediction condition), they had little difficulty in doing so. When they had the option of making inferences based on either a single-target category or multiple categories, many opted for single-category reasoning. As we predicted, however, use of single-category reasoning could be shifted quite substantially through a simple instructional manipulation. When brief instructions implied that neglect of the nontarget category could lead to a negative consequence (e.g., terminal illness or financial loss), this category was more likely to be factored into feature predictions.

The results for the standard instruction condition support the view that people often resort to a single-category heuristic when making inferences under conditions of uncertainty (e.g., Murphy \& Ross, 1994, 2005). The effect of the response-cost instructions in promoting multiplecategory reasoning, however, suggests a degree of flexibility in the way that people deal with uncertain inferences that has not been recognized previously. In previous work, a preference for single-category reasoning has been found in both artificial (e.g., Murphy \& Ross, 1994) and natural (Malt et al., 1995) categories, across a range of stimulus and task conditions. Our results suggest that this preference can be modified substantially by instructions that increase the salience of nontarget categories.

We found that the effect of response-cost instructions was unaffected by order of presentation of these instructions; response-cost instructions boosted multiple-category reasoning relative to standard instructions, whether the instructions were presented on the first or last two items. It is notable that the positive effects of the response cost, when presented for the first two items, did not facilitate multiplecategory responding on the two subsequent standard items. This may be because the response-cost instructions were tailored to the specific content of each item and were, therefore, not seen as relevant to items with a very different surface structure. In this respect, our results are similar to those of Ross and Murphy (1996), who found that people considered less likely category alternatives when predicting properties strongly associated with these alternatives, but that the same people reverted to single-category reasoning for cases where this special relationship did not exist.

\section{EXPERIMENT 2}

Experiment 1 showed that a relatively modest manipulation of the cost of inattention to less likely category alternatives increased the use of these categories in feature predictions. The response-cost instructions did not explicitly direct people to attend to the nontarget category, nor did they say anything about how information from that category should be used in feature predictions. Nevertheless, the indirect reference to the cost of ignoring the nontarget category was sufficient to shift people toward a multiple-category approach.

Experiment 2 examined the response-cost effect in more detail - in particular, the effects of the timing of the response-cost instructions relative to the categorization decisions and predictions for each item. In Experiment 1, these instructions appeared after the categories had been presented and just before people were asked to categorize and make predictions about a test exemplar. It could be argued that this timing set up a demand effect, suggesting to participants that the response-cost instructions were relevant to, and should be used in, the upcoming predictions; in other words, the response-cost effect in Experiment 1 may have been an artifact of instruction timing, rather than the result of an increase in the relative salience of the nontarget category.

Experiment 2 therefore examined whether the responsecost effect depended on the timing of cost instructions during item presentation; in this case, the instructions were presented before, rather than after, the experimental categories for each item. This should minimize any implied demand that participants should use the instructions in subsequent predictions. If the effects of response-cost instructions in Experiment 1 were an artifact of instruction timing, these effects should disappear under this new protocol. If, however, they reflect a more general process involving changes in the relative salience of the categories, we should still see a positive effect of these instructions on multiple-category predictions.

\section{Method}

Participants. Sixty-four undergraduate psychology students (36 females, 28 males; average age, 19.64 years) participated for course credit.

Materials and Procedure. These were identical to those in Experiment 1 , except that the additional response-cost instructions were presented together with the general instructions for each item before each experimental category was presented. The screen containing these instructions was visible for $6 \mathrm{sec}$. The instructions then disappeared and the categories were then presented for study. To help participants remember which category labels were associated with the low and high response-cost alternatives, these were paraphrased below each label when the category was presented (e.g., below "Terragaxis," it read "easily treatable"; below "Buragamo" it read "serious and possibly terminal"). After $8 \mathrm{sec}$, a novel test exemplar appeared and test questions were presented in the same manner as in Experiment 1. To check whether participants remembered the associations between response-cost instructions and category labels, a forced-choice memory question (e.g., "Can you remember which disease was serious and possibly terminal?" with Terragaxis and Buragamo as the response alternatives) was presented after the second response-cost item. ${ }^{3}$ 


\section{Results and Discussion}

Preliminary analyses again confirmed that item version had no effect on any of the outcome measures. All subsequent analyses were collapsed over this factor. The effectiveness of the response-cost manipulation depended on participants correctly remembering the cost instructions associated with target and nontarget categories. Most participants $(84 \%)$ remembered this association correctly for the item that was tested, confirming that they accurately encoded and retained the cost instructions.

Target category identification. The target category was identified correctly for most items in the nondivergent and divergent prediction conditions (.96 and .91, respectively). Confidence in choice of the target category was reasonably high $(M=65.48)$ and unaffected by prediction condition, response cost, or instruction order (all $F_{\mathrm{S}}<1$ ).

Feature predictions. Feature predictions were coded and analyzed in the same way as those in Experiment 1 had been. Feature prediction analyses were based only on items where the target category was identified correctly. As in Experiment 1, the proportion of multiple-category predictions in the nondivergent prediction conditions was above chance, showing that when the target category alone could not produce a clear feature prediction, people looked to information from the nontarget category $[t(30)=6.44$, $p<.001$, for standard instructions; $t(30)=5.47, p<.001$, for response cost]. Multiple-category predictions (Figure 2) were entered into a three-way ANOVA (prediction condition $\times$ instructions $\times$ instruction order) with repeated measures on the second factor. As in Experiment 1, there were fewer multiple-category responses in the divergent than in the nondivergent prediction conditions $[F(1,60)=$ 24.98, $\left.p<.001, \eta_{\mathrm{p}}^{2}=.3\right]$. When those in the divergent group failed to make multiple-category predictions, they almost always made single-category predictions (on $95 \%$ of occasions). As in Experiment 1, however, single-category reasoning was moderated by response-cost instructions $\left[F(1,60)=4.25, p=.04, \eta^{2}=.07\right]$. Figure 2 shows that when response-cost instructions were present, the proportion of multiple-category predictions in the divergent condition increased, relative to the nondivergent prediction condition. Tukey's HSD tests confirmed that people made fewer multiple-category predictions in the divergent than in the nondivergent condition when standard instructions were present ( $q=4.53, p=.02$ ), but that levels of multiple category prediction did not differ across prediction conditions when response-cost instructions were present $(q=2.26$, $p=.11$ ). There were no main effects or interactions involving the order in which the response-cost instructions (i.e., the first two items vs. the last two items) were presented. Confidence in feature predictions was moderate overall $(M=57.26)$ and unaffected by prediction condition, response cost, or instruction order (all $F_{\mathrm{s}}<2$ ).

These results replicate the main findings of Experiment 1 . We again found evidence that people often make inductive predictions on the basis of a single uncertain category. However, we also found that this strategy can be altered by relatively simple instructions that underline the cost of neglecting less likely category alternatives. Under response-cost instructions, people were more likely

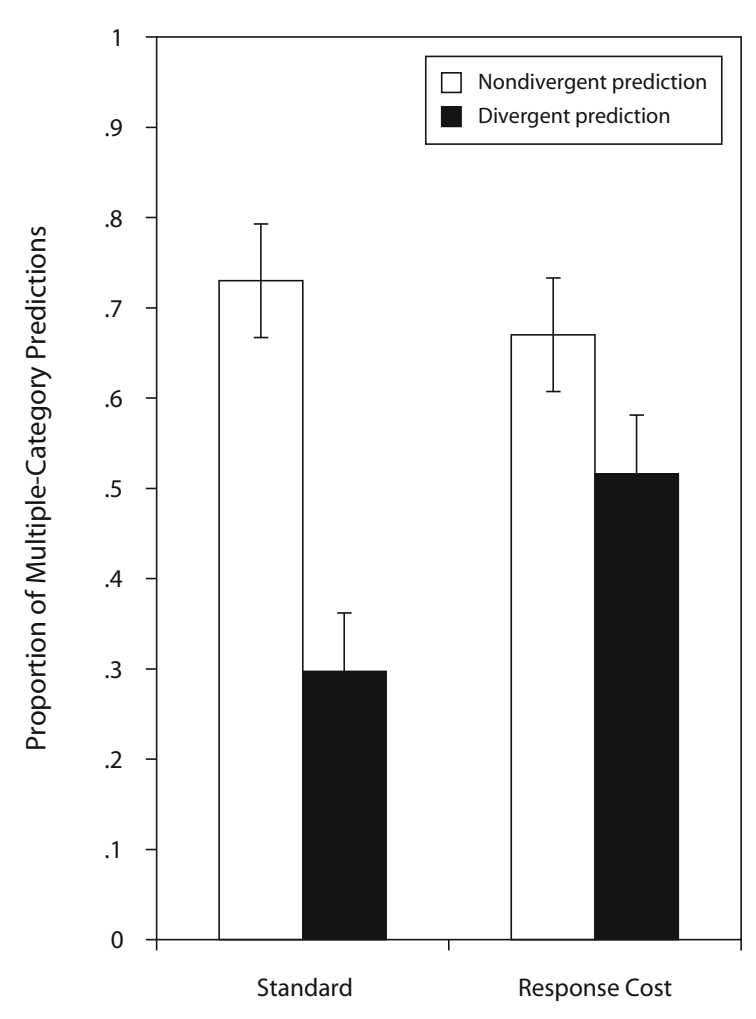

Instructions

Figure 2. Experiment 2: Mean proportion of feature predictions based on multiple categories.

to take both target and nontarget categories into account when making feature predictions. Critically, this responsecost effect was maintained when the cost instructions were presented before the category stimuli, and well before any feature predictions were required. This shows that the response-cost effects found in Experiment 1 were not simply an artifact of the timing of the cost instructions.

\section{EXPERIMENT 3}

Our argument that the relative salience of target and nontarget categories can determine whether nontargets are used in reasoning has important implications for interpreting the results of previous work in this area. In most previous studies of reasoning with uncertain categories (e.g., Murphy \& Ross, 1994), people have been asked to decide on the most likely category membership of an uncertain test exemplar before making predictions about its properties. Although this procedure has the advantage of ensuring that participants recognize the target category at the time of making an inference (those that do not are usually eliminated from analyses), the question order may be problematic in other ways. In particular, the identification of the target category just prior to making a feature prediction may draw people's attention to that category (and away from the nontarget). This may prime use of the 
target in the subsequent prediction, leading to an artifactual increase in single-category reasoning.

Supporting this account, Lagnado and Shanks (2003) have shown that categorization decisions have serious consequences for subsequent inferences. They presented people with "nonaligned" category hierarchies, in which a less probable superordinate contained a highly probable subordinate category (e.g., overall, tabloid newspapers were read more frequently than broadsheet newspapers, but the most frequently read newspaper was a particular broadsheet). When people were simply asked to learn about, and draw inferences from, the properties of the high-probability subordinate, they did so accurately. However, if they were first asked to make a superordinate categorization decision (i.e., decide which type of newspaper was more likely to be read), people subsequently overestimated the probability of subordinates within that general category and underestimated the probabilities of subordinates from alternative branches of the hierarchy. Lagnado and Shanks attributed this to a "commitment heuristic," where the act of identifying the most probable superordinate category increases confidence that this is the true category, and leads to neglect of category alternatives.

It is possible that some of the evidence for singlecategory reasoning (e.g., Murphy \& Ross, 1994) may reflect the operation of a similar mechanism, whereby identification of the target category before a property prediction increases the salience of that category relative to the nontarget. This would lead to an increased focus on the target in subsequent predictions. To examine this claim in Experiment 3, we varied the order in which categorization judgments and property predictions were made. If our account is correct, we should see more evidence of multiplecategory reasoning when people make predictions about novel exemplars before categorizing them.

A related problem is that some participants may misinterpret the prediction question as being conditional on the outcome of the categorization decision (in effect, reading the prediction question as "given that the object has feature $\mathrm{X}$ and is a member of category $Y$, what is the most likely feature?"). This again would lead to inflation of single-category responses.

These problems have been discounted in three previous examinations of the effects of question order. We would argue, however, that none of these studies has really settled the issue. Murphy and Ross (1994, Experiment 2) removed the categorization question from their procedure and still found no impact of multiple categories on feature prediction. Unfortunately, the study was limited by a relatively weak manipulation of single- and multiple-category predictions. Each approach predicted the same feature but with different levels of probability. The predicted probability difference, however, was quite small (10\%), raising doubts about whether the design was sensitive enough to discriminate between the two reasoning strategies. More recently, Murphy and Ross (2005, Experiment 4) found that question order did not have an impact on single-category predictions about more naturalistic categories (morphed images of real objects). Again, though, there is some doubt about the generality of these conclusions because of the way in which category uncertainty was manipulated. Across different trials, participants were asked to make predictions about both uncertain cases (where the test object could have been a member of at least two categories) and certain cases (where there was only a single plausible category). The presence of the certain cases may have boosted participants' confidence in their ability to identify the "correct" category in uncertain trials, leading to an inflated effect of category reasoning. Most notably, in studies that had a design similar to our own (Murphy \& Ross, 1994, Experiments 5 and 6), where single- and multiple-category approaches produced divergent feature predictions, the removal of the category question led to a marked reduction in predictions based on single categories ( $82 \%$ with question present vs. $58 \%$ with question absent).

In this experiment, we explicitly manipulated the order of test questions for each item, with some participants making categorization decisions before feature predictions (as in Experiments 1 and 2) and others receiving these questions in the reverse order. As was done in previous studies, we used items leading to either divergent or nondivergent predictions. Contrary to practice in previous studies, however, this factor was manipulated within participants, with participants completing both kinds of items. The aim was to allow for closer examination of the extent to which individual subjects consistently used multiple-category reasoning across nondivergent and divergent prediction items.

\section{Method}

Participants. Eighty first-year psychology students (46 females, 34 males; average age, 20.9 years) participated for course credit.

Design and Procedure. The experiment followed a 2 (question order) $\times 2$ (prediction condition) design with repeated measures on the last factor. Four scenarios used to explain categories and exemplar features were the same as in Experiments 1 and 2, and another four were created for this experiment (see Appendix A). The assignment of specific scenarios to nondivergent and divergent prediction conditions was counterbalanced across participants, so that each scenario appeared on an equal number of occasions in each condition.

This experiment was run in two phases. The procedure for stimulus presentation for the first phase was similar to that in Experiments 1 and 2, except that participants completed a total of eight items presented in two blocks of four. One block contained nondivergent prediction items and the other contained divergent prediction items. Block order was counterbalanced across participants. The blocks were separated by an unrelated 10-min filler task (rating the typicality of members of social/occupational and food categories). Half the participants received test questions in the same order as in the previous experiments (with categorization followed by feature predictions), whereas for the remainder this order was reversed.

\section{Results}

Preliminary analyses confirmed that, with one exception (see feature prediction analyses for details), the scenario version used to describe categories and exemplars had no effect on performance. Identification of target categories and feature predictions was unaffected by the order in which participants completed nondivergent and divergent prediction blocks. Subsequent analyses were collapsed over these factors.

Target category choices. The target category was identified correctly in most cases $(M=.95)$. Identification was reliably better for divergent prediction items $(M=$ 
.98) than for nondivergent items $(M=.92)[F(1,72)=$ $\left.10.00, p=.002, \eta_{\mathrm{p}}^{2}=.22\right]$. In general, people were moderately certain about identification of the target category $(M=68.7)$, but were more certain about these decisions for divergent prediction items $(M=70.78)$ than for nondivergent prediction items $(M=66.7)[F(1,72)=5.9, p=$ $\left..02, \eta_{\mathrm{p}}^{2}=.14\right]$. Neither target category identification nor identification confidence were affected by question order (all $F \mathrm{~s}<1.5$ ).

Feature predictions. Feature predictions (see Figure 3) were coded and analyzed in the same way as in the previous experiments. Items were included in the feature prediction analyses only when the target category for that item was correctly identified. Data from 2 participants were dropped from the analysis because the participants failed to identify the correct target category for any items in one of the prediction conditions. Nondivergent prediction performance for one scenario (planet type) was substantially lower ( $M=.25$ multiple-category predictions) than in the other seven scenarios, so predictions based on this scenario were not included in the analysis. ${ }^{4}$

Figure 3 shows that the level of multiple-category reasoning was lower than in Experiments 1 and 2. Levels of multiple-category prediction in the nondivergent prediction, in particular, were below those found previously. This is most likely due to the within-participants mixture of nondivergent and divergent prediction items. Apart from increasing task duration and overall cognitive demand, this mixture of items could require switching of reasoning strategies across items (e.g., from single-category on divergent items to multiple-category on nondivergent items). Our data suggest that some people had difficulty with such strategy switching. Participants attempting to use a singlecategory strategy for the nondivergent items would have led to a higher rate of "other" feature predictions; this is reflected in the data. In the nondivergent condition for this experiment, the mean rate of "other" predictions was .32, compared with a mean rate of .22 in Experiments 1 and 2.

Nevertheless, the proportion of multiple-category predictions for nondivergent items was still well above chance $[t(77)=6.39, p<.001]$. Moreover, we found fewer multiple-category predictions for divergent than for nondivergent items $\left[F(1,70)=13.94, p<.001, \eta_{\mathrm{p}}^{2}=\right.$ $.19]$. When they did not make multiple-category predictions, participants made single-category predictions for divergent items (on $90 \%$ of occasions). As predicted, however, this effect interacted with the order of test questions $\left[F(1,70)=4.09, p=.04, \eta_{\mathrm{p}}^{2}=.06\right]$. Figure 3 shows that when predictions were made before categorization, the proportion of multiple-category predictions in the divergent condition increased.

People were generally more confident in their feature predictions for divergent items $(M=57.6)$ than for nondivergent items $(M=51.3)[F(1,70)=10.04, p=.002$, $\left.\eta_{\mathrm{p}}^{2}=.22\right]$. No other significant main effects or interactions for feature predictions or feature confidence were found.

\section{Discussion}

Despite lower overall levels of multiple-category responding, this experiment yielded a number of important

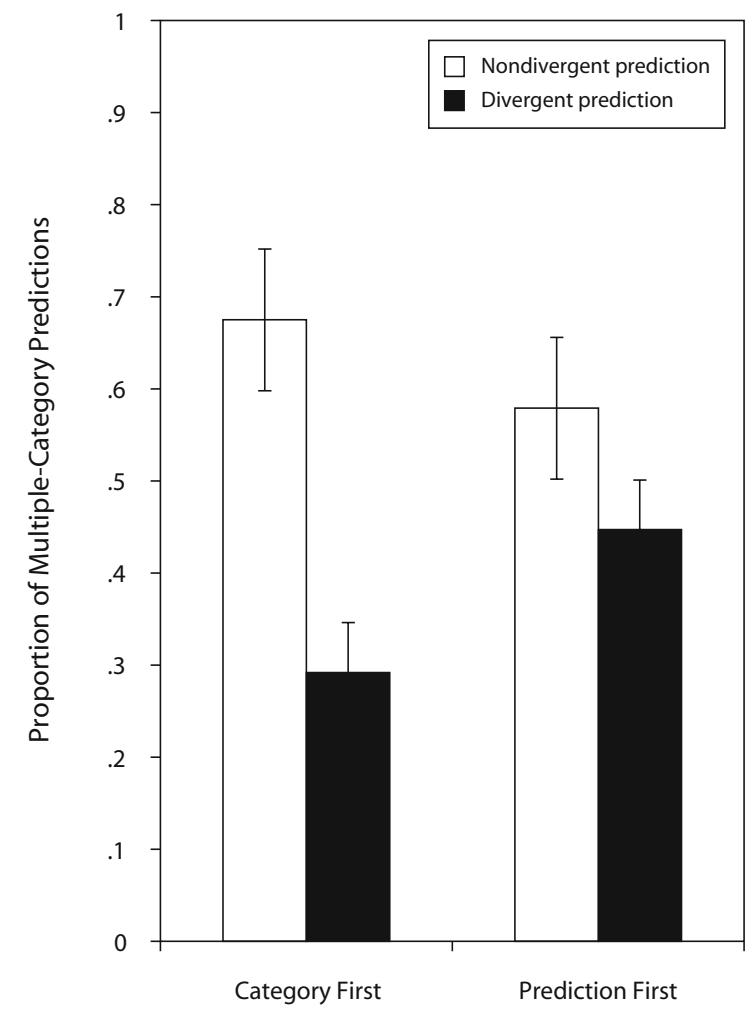

Figure 3. Experiment 3: Mean proportion of feature predictions based on multiple categories.

findings. As in our previous experiments, multiple-category reasoning was less common for the divergent prediction items than for the nondivergent ones. Once again, many people made predictions based only on consideration of the target category for divergent prediction items. As predicted, however, the order in which people made feature predictions and category membership judgments about novel exemplars had a substantial impact on reasoning. When people made feature predictions about a novel exemplar before deciding on its category membership, they were more likely to reason using multiple categories.

In most previous work on reasoning under category uncertainty (e.g., Murphy \& Ross, 1994), feature inferences were made only after novel exemplars were categorized. Our findings indicate that this may have artificially elevated levels of single-category reasoning. When predictions follow category judgments, people may use singlecategory reasoning not because it is a default strategy but because the categorization question itself draws attention to the target category.

It is important to note that this account is inconsistent with the Bayesian approach to reasoning under uncertainty. From the Bayesian standpoint, it is no more rational to focus on a single category because you have already declared it as the most likely alternative than it is to use it as a heuristic shortcut to avoid complex computation. Although our question order data suggest that previous accounts of 
single-category reasoning may be incorrect, they also suggest that there are factors having an impact on reasoning with uncertain categories that lie outside the bounds of theories such as Anderson's (1991) Rational model.

A potential objection to our reversal of the standard question order is that when feature prediction precedes categorization we cannot be certain that people are aware of the correct target category when making predictions. The present data suggest that this was unlikely to be the case. In the prediction-first condition, the accuracy of target category identification for divergent prediction items was very high ( $M=98 \%$ correct $)$ and was comparable to that in the category-first condition ( $M=99 \%$ correct). Hence, it seems likely that those in the prediction-first condition would have been able to accurately distinguish between the target and nontarget categories before making an explicit categorization judgment.

\section{GENERAL DISCUSSION}

These experiments examined how people make feature predictions about exemplars whose category membership is uncertain. The main question was whether people consider multiple category alternatives when making predictions, or whether they base their predictions only on the most likely category. Like the authors of many previous studies (Malt et al., 1995; Murphy \& Ross, 1994, 2005; Ross \& Murphy, 1996), we found that people do not always consider multiple categories when making feature predictions. Unlike previous authors, however, we found that the extent to which people engage in multiple-category reasoning was strongly affected by instructional and task factors that increase the relative salience of the nontarget category. A brief instructional manipulation that highlighted the cost of neglecting less likely category alternatives led to a substantial increase in multiple-category reasoning (Experiments 1 and 2). Multiple-category reasoning was also more likely to occur when people were not forced to classify test exemplars before they made feature predictions (Experiment 3 ).

Much of the previous work in this area has portrayed single-category reasoning as a pervasive heuristic employed in most cases of feature prediction with uncertain categories. Our results show that single-category reasoning may not be as pervasive as has been claimed. Rather than being a default approach, single-category reasoning may become the dominant reasoning strategy only under relatively constrained conditions.

\section{Comparison With Previous Research}

Our findings on the effects of "response-cost" instructions on multiple-category reasoning are reminiscent of other findings in experiments that have tried to overcome the negative effects of cognitive heuristics in studies of judgmental anchoring (Mussweiler, Strack, \& Pfeiffer, 2000), forecasting (Lovallo \& Kahneman, 2003), social judgment (Lord et al., 1984), and probability judgment (Lagnado \& Sloman, 2004). In each of these cases, the main focus has been on improving judgment and pre- diction by getting people to consider multiple alternate hypotheses about uncertain situations. Mussweiler et al., for example, demonstrated that the magnitude of an anchoring effect shown by car experts estimating the value of a used car was reduced simply by asking the experts to list anchor-inconsistent arguments. A similar remedy was prescribed by Lovallo and Kahneman to overcome a tendency in judgmental forecasting to focus solely on the particular problem at hand (an "inside view") at the expense of thinking about the current problem in the context of similar situations that we might have encountered or know about (an "outside view"). In all of these cases, making participants aware of other potential influences on the outcome of events improved judgments and predictions (cf. Lagnado \& Sloman, 2004).

We have interpreted the question-order effect in terms of changes to the relative salience of target and nontarget categories, with the explicit categorization decision causing people to direct attention to the target category and priming its use in subsequent predictions. In broad terms, this effect resembles the "commitment effect" in category hierarchies reported by Lagnado and Shanks (2003), whereby focusing on one part of a category hierarchy in a decision causes a bias toward that same branch of the hierarchy in subsequent decisions. Lagnado and Shanks, however, offered an explanation of this effect that is somewhat different from our account of the effects of question order. They saw the commitment effect as driven by an inflation in confidence that the first decision is "correct" and hence that the second decision should be conditional upon the first. This explanation seems unlikely to apply to our findings concerning question order. The Lagnado and Shanks account implies that people should be more confident in their predictions when they have previously identified the target category. However, we found no differences in prediction confidence as a function of question order.

The levels of single-category reasoning that we observed in our divergent prediction conditions were generally lower than those found in corresponding conditions used in previous work. In Experiment 3, for example, in the divergent condition with category questions first, people chose the single-category feature around $70 \%$ of the time. This is lower than the corresponding result (82\%) reported by Murphy and Ross (1994, Experiment 6), who also used a feature-choice measure of reasoning. There are a number of task and procedural differences between our experiments and previous work that may have contributed to these different levels of single- and multiple-category inference. First, only two categories were presented in each prediction task. In previous work (e.g., Murphy \& Ross, 1994), up to four alternative categories were present when people made category judgments and feature predictions. It is possible that being exposed to a wider range of alternative categories increases the perceived complexity of factoring these into a prediction, leading to greater use of a single-category heuristic or guessing. However, although multiple categories were present in most previous studies, in most cases only two categories were relevant to a given prediction, as was the case in the 
present experiments. Hence, it seems unlikely that this factor alone would have produced a marked increase in multiple-category reasoning.

A second way in which our procedure differed from past work was in the use of text descriptions of exemplar features. In contrast, Murphy and Ross have often used geometric exemplars varying in shape and color. There have been some suggestions that people may treat shapecolor combinations as integral stimuli (Love \& Markman, 2003). It may require some effort to parse such stimuli into their components, making the assessment of the feature frequencies more difficult than in our text descriptions. These additional computational demands may have made the use of a simplifying heuristic, such as single-category reasoning, more attractive.

Although we do not discount the impact of these factors, the general picture that arises from comparisons with previous studies is that single-category reasoning is more likely to be found when the processing demands of task are considerable (e.g., when people have to encode the exemplars from several categories, and/or do some extra computation to work out feature frequencies). Conversely, when comparison of feature frequencies within and between categories is relatively straightforward, and when there are only a small number of categories to consider, people seem capable of making feature predictions based on information from multiple categories.

\section{Implications for Theories of Reasoning Under Uncertainty}

As outlined at the beginning of this article, one explanation for single-category reasoning is that people do not understand the conditional nature of making predictions about objects with uncertain category membership. Our data do not support this account. When it was not possible to make a clear prediction from a single category, most participants had little difficulty in integrating information from the less likely category into their predictions. Together with the response-cost conditions in Experiments 1 and 2, and prediction-first conditions in Experiment 3, according to these data, people are capable of understanding that uncertainty about an object's category membership means that alternative categories should be considered when property predictions are made.

The two-stage explanation outlined by Ross and Murphy (1996) assumes that people are capable of grasping the relevance of category uncertainty for prediction, but that information about the nontarget is rarely accessed at the prediction stage. Our results suggest that an important modification to this account is necessary. We have shown that factors that affect the relative salience of the target and nontarget categories at the categorization stage also affect whether the nontarget categories are used at the prediction stage. Drawing attention to the nontarget category through the response-cost instructions can boost use of the nontarget category in subsequent feature predictions. Reducing attention to the target category via elimination of explicit categorization before prediction also facilitates multiple-category reasoning.
In the present experiments, we tried to ensure that people used categories to make predictions rather than using a noncategorical approach (e.g., feature conjunction) by making it impossible to arrive at a prediction by just looking at feature pairings across exemplars. Another possibility, however, is that participants ignored both the categories and the given feature, and made predictions based only on overall base rates of feature values (e.g., in Table 1, divergent condition, there are clearly more exemplars with "sore gums" overall). Several results, however, indicate that participants did not use this noncategorical strategy. First, if they did use this approach, similar levels of multiple-category prediction should have been found in the nondivergent and divergent conditions. However, a main effect difference in multiple-category predictions in favor of the nondivergent condition was found in every experiment. Second, it is hard to see how just attending to overall feature base rate would explain the changes in prediction patterns produced by the response-cost and question-order manipulations.

\section{Implications for Bayesian Models of Inference}

In the response-cost conditions of Experiments 1 and 2, and in the prediction-first condition of Experiment 3, many people used multiple categories when making feature predictions. In this respect they were behaving in a way that more closely approximates Bayesian prescriptions like Anderson's (1991) Rational model.

We would stop short, however, of concluding that approaches like the Rational model give an accurate description of the way that people carry out reasoning under category uncertainty. One reason for being cautious about such conclusions is that the Rational model provides no explanation how and why people would employ single- or multiple-category reasoning flexibly across different task and prediction contexts. Another reason for caution is that, contrary to the Rational model, individual participants were often inconsistent in their application of multiplecategory reasoning across items. Participants were classified as making consistent use of multiple-category reasoning if their proportions of multiple-category responses were above the median within given experimental conditions for both nondivergent and divergent prediction items. Even in the prediction-first condition of Experiment 3 , for example, only a minority of participants $(30 \%)$ consistently made multiple-category predictions for both nondivergent and divergent items.

In sum, these experiments show that, under the right conditions, participants, when making predictions, give some consideration to less likely category alternatives. This does not necessarily imply that people are following a Bayesian prescription.

\section{Conclusion}

Previous work has suggested that when people make predictions about exemplars whose category membership is uncertain, they usually ignore uncertainty and consider only the most probable category. Although we also found some evidence of single-category reasoning, 
we have shown that this heuristic can be overcome by allowing people to make predictions about a novel exemplar before categorizing it and by informing people about the negative consequences of inattention to less probable alternatives. Our experiments suggest that the problem of single-category reasoning may have been exaggerated in previous work on reasoning under uncertainty. People appear capable of applying either singleor multiple-category approaches to feature prediction as demanded by the specific task and decision context. Future work should therefore be directed toward a more complete specification of the conditions that promote single- and multiple-category inference. Such data will inform and constrain the development of more detailed process models of property inference when category membership is uncertain.

\section{AUTHOR NOTE}

This work was supported by Australian Research Council Discovery Grant DP0770292 to the authors. We thank John Coley and Carissa Bonner for their valuable comments on this research, and Carissa Bonner, Brooke Hahn, and Tamara Cavenett for their assistance with data collection and comments on earlier drafts. Correspondence concerning this article should be addressed to B. K. Hayes, School of Psychology, University of New South Wales, Sydney, NSW 2052, Australia (e-mail: b.hayes@unsw.edu.au).

\section{REFERENCES}

ANDERson, J. R. (1991). The adaptive nature of human categorization. Psychological Review, 98, 409-429.

GLUCK, M. A., \& BowER, G. H. (1988). From conditioning to category learning: An adaptive network model. Journal of Experimental Psychology: General, 117, 227-247.

HaYes, B. K., RuthVEN, C., \& Newell, B. R. (2007). Inferring properties when categorization is uncertain: A feature-conjunction account. Proceedings of the 29th Annual Conference of the Cognitive Science Society (pp. 209-214). Mahwah, NJ: Erlbaum.

KoEHLER, D. J. (1994). Hypothesis generation and confidence in judgment. Journal of Experimental Psychology: Learning, Memory, \& Cognition, 20, 461-469.

Lagnado, D., \& Shanks, D. R. (2003). The influence of hierarchy on probability judgment. Cognition, 89, 157-178.

LaGnado, D., \& Sloman, S. A. (2004). Inside and outside probability judgment. In D. J. Koehler \& N. Harvey (Eds.), The Blackwell handbook of judgment and decision making (pp. 157-176). Malden, MA: Blackwell.

Lord, C. G., Lepper, M., \& Preston, E. (1984). Considering the opposite: A corrective strategy for social judgment. Journal of Personality \& Social Psychology, 47, 1231-1243.

Lovallo, D., \& Kahneman, D. (2003). Delusions of success. Harvard Business Review, 81, 57-63.

Love, B. C., \& Markman, A. B. (2003). The non-independence of stimulus properties in human category learning. Memory \& Cognition, 31, 790-799.

Malt, B. C., Ross, B. H., \& Murphy, G. L. (1995). Predicting features for members of natural categories when categorization is uncertain Journal of Experimental Psychology: Learning, Memory, \& Cognition, 21, 646-661.

Markman, A. B., Baldwin, G. C., \& Maddox, W. (2005). The interaction of payoff structure and regulatory focus in classification. Psychological Science, 16, 852-855.

McKenzIE, C. R. M. (1998). Taking into account the strength of an alternative hypothesis. Journal of Experimental Psychology: Learning, Memory, \& Cognition, 24, 771-792.
Murphy, G. L., \& Ross, B. H. (1994). Predictions from uncertain categorizations. Cognitive Psychology, 24, 148-193.

Murphy, G. L., \& Ross, B. H. (2005). The two faces of typicality in category-based induction. Cognition, 95, 175-200.

MurPHY, G. L., \& Ross, B. H. (2007). Use of single or multiple categories in category-based induction. In A. Feeney \& E. Heit (Eds.), Inductive reasoning: Experimental, developmental and computational approaches. New York: Cambridge University Press.

MurPhy, G. L., \& Ross, B. H. (2009). Induction with uncertain categories. Unpublished manuscript.

Mussweiler, T., Strack, F., \& Pfeiffer, T. (2000). Overcoming the inevitable anchoring effect: Considering the opposite compensates for selective accessibility. Personality \& Social Psychology Bulletin, 26, 1142-1150.

Osherson, D. N., Smith, E. E., Wilkie, O., López, A., \& Shafir, E. (1990). Category-based induction. Psychological Review, 101, 53-97.

RIPS, L. J. (1975). Inductive judgments about natural categories. Journal of Verbal Learning \& Verbal Behavior, 14, 665-681.

Ross, B. H., \& MURPHY, G. L. (1996). Category-based predictions: Influence of uncertainty and feature associations. Journal of Experimental Psychology: Learning, Memory, \& Cognition, 22, 736-753.

Ross, B. H., \& Murphy, G. L. (1999). Food for thought: Crossclassification and category organization in a complex real-world domain. Cognitive Psychology, 38, 495-553.

SLOman, S. A. (1993). Feature-based induction. Cognitive Psychology, 25, 231-280.

Tenenbaum, J. B., Kemp, C., \& Shafto, P. (2007). Theory-based Bayesian models of inductive reasoning. In A. Feeney \& E. Heit (Eds.), Inductive reasoning (pp. 167-204). New York: Cambridge University Press.

Trope, Y., \& MACKIE, D. (1987). Sensitivity to alternatives in social hypothesis-testing. Journal of Experimental Social Psychology, 23, 445-459.

Tversky, A., \& Kahneman, D. (1973). Availability: A heuristic for judging frequency and probability. Cognitive Psychology, 5, 207-232.

TVersky, A., \& Kahneman, D. (1981). The framing of decisions and the psychology of choice. Science, 211, 453-458.

\section{NOTES}

1. The two prediction conditions were constructed so that the probability of identifying the target category given a particular feature was similar for each. For example, in Table 1 the probability of identifying the target category given "headache" was $6 / 10=.6$ for the nondivergent prediction condition and 7/11 $=.64$ for the divergent condition. We checked whether this small objective difference in probability affected participants' ability to correctly identify the target category in each condition. There were no differences between prediction conditions in accuracy of target category identification in any experiment.

2. It is also possible that some people in the nondivergent condition tried to apply a single-category strategy, leading them to the two possible feature choices of "sore gums" and "rash." If some guessed the first alternative, this would have been coded (erroneously) as a "multiplecategory" response. To check this, we compared the proportion of multiple-category responses in the nondivergent condition against a more stringent chance value of .5. Again, responses in both responsecost conditions were above chance (both $p \mathrm{~s}<.05$ ), indicating that these responses predominantly reflected multiple-category reasoning. This was also found to be the case for both response-cost conditions in Experiment 2 and for the category-first condition in Experiment 3.

3. No memory question was presented for the first response-cost item, because we felt that this could artifactually increase attention to these instructions on the subsequent item.

4. This appeared to be an effect of prior knowledge/beliefs about relations between the features in this item. When asked to predict the surface terrain of an extraterrestrial planet, given information about its atmosphere, many people judged a feature that appeared in only a small number of exemplars (frozen) to be more likely than the features that were predicted by a multiple-category approach in either version of the stimuli (desert, mountainous). 
APPENDIX A

Instructions for Category Scenarios (Standard Condition)

\begin{tabular}{ll}
\hline Scenario & \multicolumn{1}{c}{ Instructions } \\
\hline Disease & Imagine that you are a trainee medical doctor learning about the characteristics of differ- \\
& ent diseases. In this task you are going to learn about two diseases called Terragaxis and \\
& Buragamo. To help you learn about the diseases we will show you the symptom profiles \\
& of eight patients with Terragaxis and eight patients with Buragamo. Each patient will have \\
& two symptoms.
\end{tabular}

Chemical assay In this task we would like you to imagine that you are working for a company that carries out chemical analyses of newly mined minerals. You are now going to learn about the chemical components of two types of minerals, called Xamapol and Zindapod. To help you learn about these minerals we will show you the chemical components of eight samples of Xamapol and eight samples of Zindapod.

Immigration In this task you need to imagine that you are training to work in the area of Australian Customs and Immigration, assessing whether or not to grant business visas to applicants. One part of the training for this job involves learning the previous travel patterns of visa applicants from different countries. Knowing about such travel patterns has been found to be helpful in predicting outcomes like who overstays their visa. You are now going to learn about the previous travel patterns of applicants from two countries, which we will call Country Alpha and Country Beta. To help you learn we will show you the countries that have been previously visited by eight applicants from Country Alpha and eight applicants from Country Beta.

Car defects In this task you need to imagine that you are working in the quality control section of a large automobile company. Two new models of cars manufactured by your company, called Zeparos and Catapults, have just been tested in a 6-month quality control trial. You are now going to learn about the patterns of faults that have been reported for the two kinds of models. To help you learn about these patterns we will show you the results of tests on eight Zeparos and eight Catapults.

\section{Experiment 3 Only}

Pre-schools In this task you should imagine that you are an Educational psychologist learning about the drawing ability of children from two different pre-schools. Two different pre-schools, Happy Daze Pre-school and Little Blossoms Pre-school were surveyed. You are now going to learn about the kinds of drawings done by children at each school. To help you learn we will describe the drawings done by eight children from Happy Daze Pre-school and eight children from Little Blossoms Pre-school. Each description will tell you two things about each child's drawing.

Plants In this task you should imagine that you are a botanist learning about two recently discovered plant species. The two types of plants are called Helenium and Bouvardia. You are now going to learn about the characteristics of these plants. To help you learn we will describe the features of eight samples of Helenium and eight samples of Bouvardia. Each description will tell you two things about each plant.

Resorts In this task you should imagine that you are deciding where to take your annual holiday. You are considering two resorts, called Club Aus and GetALife. Before you decide on your destination you think it's a good idea to check reviews of each resort on a travel website. To help you with your decision we will give you samples from eight reviews of Club Aus and eight reviews of GetALife. Each sample will tell you two things (best feature, worst feature) about each resort.

Planet classes In this task you should imagine that you are an astronomer learning about the characteristics of two kinds of planets that have been discovered in another solar system. The two groups of planets are called Class E and Class K. To help you learn each class we will describe some of the characteristics of eight Class E planets and eight Class K planets. Each description will tell you two things about each planet. 
APPENDIX B

Additional Instructions for Response-Cost Conditions

Scenario Instructions

Disease

Please Note: The [nontarget disease] is very serious, and possibly terminal. The [target disease] is a relatively minor, easily treatable illness.

Chemical assay Please Note: The [nontarget mineral] is very rare and has the potential to be sold for very high profits. The [target mineral] is relatively common and not so profitable.

Immigration Please Note: Some people from [nontarget country] have been suspected of involvement in terrorist activities. No such reports have been made about people from the [target country].

Car defects Please Note: During testing some cars from the [nontarget model] were involved in on-road breakdowns that have the potential to cause very serious injuries to car occupants. No such reports were made for the [target model].

(Manuscript received June 13, 2008;

revision accepted for publication March 4, 2009.) 